\title{
Quantum Dot Lasers Subject to Polarization-Rotated Optical Feedback
}

Lin, Hong; Hong, Yanhua; Ourari, Salim; Huang, Tianyao; Yang, Chen

\section{IEEE Journal of Quantum Electronics}

\author{
DOI: \\ 10.1109/JQE.2019.2953518
}

Published: 01/02/2020

Peer reviewed version

Cyswllt i'r cyhoeddiad / Link to publication

Dyfyniad o'r fersiwn a gyhoeddwyd / Citation for published version (APA):

Lin, H., Hong, Y., Ourari, S., Huang, T., \& Yang, C. (2020). Quantum Dot Lasers Subject to

Polarization-Rotated Optical Feedback. IEEE Journal of Quantum Electronics, 56(1), [2000308]. https://doi.org/10.1109/JQE.2019.2953518

\footnotetext{
Hawliau Cyffredinol / General rights

Copyright and moral rights for the publications made accessible in the public portal are retained by the authors and/or other copyright owners and it is a condition of accessing publications that users recognise and abide by the legal requirements associated with these rights.

- Users may download and print one copy of any publication from the public portal for the purpose of private study or research.

- You may not further distribute the material or use it for any profit-making activity or commercial gain

- You may freely distribute the URL identifying the publication in the public portal ?
}

Take down policy

If you believe that this document breaches copyright please contact us providing details, and we will remove access to the work immediately and investigate your claim. 


\title{
Quantum Dot Lasers Subject to Polarization- Rotated Optical Feedback
}

\author{
Hong Lin, Yanhua Hong, Salim Ourari, Tianyao Huang, and Chen Yang
}

\begin{abstract}
Dynamics of a multimode quantum dot laser with polarization-rotated optical feedback is investigated in a wide range of bias current. The results reveal that the laser is more sensitive to optical feedback when the polarization of feedback beam is rotated a large angle from the original polarization, which is different from the results in DFB lasers. Anticorrelated fluctuations between orthogonal polarizations are observed in a certain range of selected polarizations. Accordingly, dynamics in the total power can be weaker than that in a selected polarization. The anticorrelated polarization dynamics may be related to different polarizations of longitudinal modes.
\end{abstract}

Index Terms-Optical feedback, polarization, quantum dot lasers

\section{INTRODUCTION}

$\mathrm{U}$ SING multiple layers of nanostructures as active medium, quantum dot lasers (QDLs) have advantages over bulk and quantum-well counterparts in many respects such as threshold current, temperature stability, and beam quality [1][3]. These advantages make quantum dot lasers promising optical transmitters for data communications, especially in short-reach communication links. Because of finite intraband relaxation time, a QDL can exhibit excited-state (ES) transition in addition to ground-state (GS) transition [4]. These two emissions can occur exclusively or simultaneously. Accordingly, a QDL can emit two wavelengths that differs several tens of nanometers, thus being possible for terahertz applications.

It is well known that bulk and quantum-well semiconductor lasers are sensitive to optical feedback. Optical

Manuscript received August 16, 2019. This work was supported in part by a Phillips Faculty Fellowship from Bates College.

H. Lin is with the Department of Physics and Astronomy, Bates College, Lewiston, ME 04240, USA (phone: 207-786-6320; fax: 207-786-8334; email: hlin@ bates.edu).

Y. Hong is with the School of Computer Science and Electronic Engineering, Bangor University, Wales, LL57 1UT, UK (email: y.hong@bangor.ac.uk).

S. Ourari was with Bates College, Lewiston, ME 04240 USA. He is now with the Department of Electrical Engineering, Princeton University, Princeton, NJ 08544 USA (e-mail: sourari@ princeton.edu).

T. Huang was with Bates College, Lewiston, ME 04240 USA. He is now with the Department of Electrical Engineering, Northwestern University, Evanston, IL 60208 USA (email: Maxhuang2024@u.northwestern.edu).

C. Yang is with Bates College, Lewiston, ME 04240 USA (e-mail: cyang@bates.edu). feedback can result in threshold reduction, linewidth broadening or narrowing, low frequency fluctuations, bistability, antiphase fluctuations, and chaos [5]-[12]. While being unwanted in applications requiring stable lasers, optical feedback has proven to be useful in chaos-based security communications [13], random number generation [14], and photonic generation of microwave signals [15]. Compared to quantum-well lasers, QDLs operating in GS emission are less sensitive to optical feedback [16]-[22]. The weak sensitivity is attributed to smaller linewidth enhancement factor [16], [22], stronger damping in relaxation oscillation [17]-[22], and weaker phase-amplitude coupling [21]. This low sensitivity to optical feedback can be exploited for isolator-free systems. On the contrary, the ES emission of QDLs can be much more sensitive to optical feedback due to smaller damping rate, and its route to chaos is different from that of the GS emission [23], [24]. A recent paper reported that in QDLs directly grown onto silicon, resistance to optical feedback increases when the QDL transits from the dual emission regime to the sole ES regime [25]. It is also demonstrated that modal powers display chaotic antiphase oscillations while the total output power remains constant [26].

In the study of optical feedback, polarization of optical feedback has attracted a great deal of attention. Polarized optical feedback can eliminate polarization switching in VCSELs [27]. Dynamics of polarized optical feedback is studied experimentally and theoretically in both single- and multi-transverse mode regimes of VCSELs [28], [29]. Early motivation for investigating polarization-rotated feedback was related to possible advantages of chaos generated by polarization-rotated feedback for secure communication systems [30]-[33]. Polarization-rotated feedback can induce square wave pulsations [33], result in broadband power spectrum [14], and conceal time-delay signatures [35], [36]. It is shown that when the polarization of feedback beam is rotated by $90^{\circ}$ relative to the original polarization of the laser (often referred as TM-mode feedback while the original polarization is named TE-mode), neither threshold reduction nor wavelength shift occurs [30]. The TM-mode oscillation may delay from the TE-mode oscillation by the round-trip time in the external cavity [30] or manifest antiphase oscillation [33]. However, prior studies on QDLs with optical feedback have focused on isotropic optical feedback, which means that the polarization of feedback beam is not changed from the original polarization. Little study was done on the response of QDLs to polarization-rotated optical feedback. 
In this paper, we will present an experimental study of the behaviors of a multimode QDL subject to polarization-rotated feedback. Our study unveils that the QDL is more sensitive to polarization-rotated feedback in a certain parameter region. Polarization dynamics of the QDL is explored as well. To our knowledge, this was the first investigation on QDL dynamics triggered by polarization-rotated optical feedback. In Section II, we will describe the experimental setup and static features of the QDL without and with optical feedback. Sec. III focuses on laser dynamics, and Sec. IV is for discussion and conclusion.

\section{Power AND SPECTRAL FEATURES OF THE QDL}

The experimental setup is shown in Fig. 1. A commercial quantum dot laser (QD Lasers, QLF133A-P5) is used in our experiment. With Fabry-Perot (F-P) cavity, the quantum dot laser (QDL) operates at $1.3 \mu \mathrm{m}$. Its bias current is controlled with a low-noise current source (Yokogawa GS200) and its temperature is stabilized within $0.01^{\circ} \mathrm{C}$ (ILX Lightwave LDT5412). The output beam is collimated with a collimating lens (CL) and split by a non-polarizing beam splitter (BS). The power reflectivity of BS is measured to be $78.7 \%$. A wellaligned mirror (M) forms an external cavity and provides optical feedback to the QDL, where M is aligned to maximize output power near the threshold. For most results reported in this paper, the distance between $\mathrm{M}$ and the QDL is around 32 $\mathrm{cm}$. The power reflectivity of $\mathrm{M}$ is $89 \%$ for $1.3 \mu \mathrm{m}$. The power reflectivity of the external cavity is changed with a variable neutral density filter (NDF). A quarter-wave plate (QWP) is inserted in the external cavity to change the polarization of the feedback beam. The polarization of the QDL is examined with a linear polarizer (LP). The solitary QDL is linearly polarized. With the half-wave plate (HWP) behind the collimating lens, the polarization of the output beam is set along the direction parallel to the optical table, which is named the x-direction. When the quarter-wave plate makes an angle $\theta$ with the $\mathrm{x}$ direction, the feedback beam will be rotated an angle $\theta_{\mathrm{p}}$ after a round trip in the external cavity, where $\theta_{\mathrm{p}}=2 \theta$. The angle $\theta_{\mathrm{p}}$, termed polarization angle, is illustrated in the $x-y$ coordinate system at the upper left corner of Fig. 1. The xy plane represents the cross section of the laser beam. The light propagates in the $\mathrm{z}$-direction, where the $\mathrm{x}, \mathrm{y}$, and $\mathrm{z}$ axes follow the right-hand rule. When $\theta_{\mathrm{p}}$ is between $90^{\circ}$ and $180^{\circ}$, the angle between the rotated polarization and the original polarization is $180^{\circ}-\theta_{\mathrm{p}}$. The transmitted light through the BS is sent to a multimode fiber coupler $(1 \times 2 \mathrm{FC})$ that splits the beam into two parts, which allows us to measure two quantities (e. g., output power and optical spectrum) at the same time. The output power and optical spectrum are measured with a power meter (Anritsu ML9001A) and an optical spectrum analyzer (Agilent 8614B, resolution $0.06 \mathrm{~nm}$ ), respectively. The feedback induced dynamics is recorded with fast detectors (New Focus 1554-B-50, $12 \mathrm{GHz}$ ) connected to a power spectrum analyzer (Anritsu MS2667C, $9 \mathrm{kHz}$ to $30 \mathrm{GHz}$ ) and a digital oscilloscope (Tektronix TDS7404, 4 GHz), respectively. For polarization resolved measurements, a half- wave plate combined with a polarizing beamsplitter are used. These optical elements, along with the equipment for dynamics measurement, are not shown in Fig. 1.

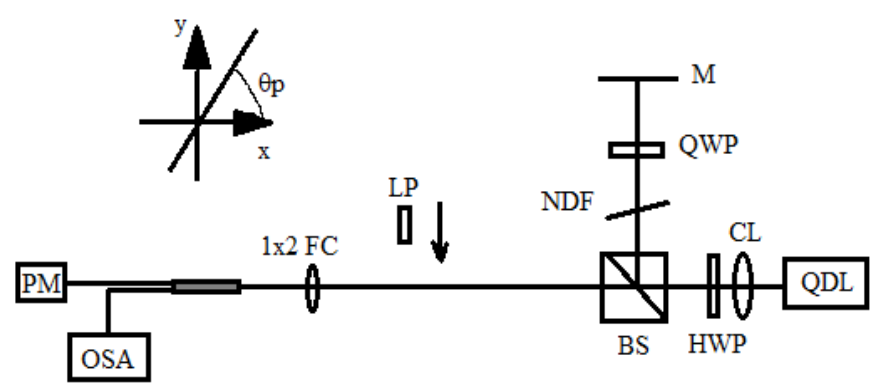

Fig. 1. Experimental setup, where QDL stands for quantum dot laser, CL stands for collimating lens, HWP stands for half-wave plate, BS for nonpolarizing beamsplitter, NDF for neutral density filter, QWP for quarter-wave plate, M for mirror, LP for linear polarizer, 1x2 FC for one-to-two fiber coupler that splits the input into two parts sending to two apparatus (e. g., PM and OSA, respectively, where PM is a power meter and OSA represents optical spectrum analyzer). The xy coordinate system represents the plane perpendicular to the direction of light propagation, with light propagating in the z-direction (out of the page). $\theta_{\mathrm{p}}$ is the angle between the polarization of feedback beam and the $\mathrm{x}$-axis, measured counterclockwise from the $\mathrm{x}$-axis.

The power-current (P-I) curves of the QDL without and with optical feedback are shown in Fig. 2, where the upper row is for a wide range of bias current, and the lower row is zoomed in near threshold. Threshold current is found by using the conventional method, that is, using linear portion of the curve to identify the intercept with the horizontal axis. The threshold current of the solitary QDL is $6.22 \mathrm{~mA}$. The feedback is termed isotropic optical feedback when the polarization of feedback beam is the same as the original polarization $\left(\theta_{\mathrm{p}}=0\right)$. The threshold is reduced to $5.77 \mathrm{~mA}$ by isotropic feedback (Fig. 2c), which is expected since optical feedback increases threshold gain [5]. However, the power is less than that of the solitary QDL when the current is $10 \mathrm{~mA}$ and higher, thus the slope of the P-I curve is less than that of the solitary QDL (Fig. 2a). Given that optical feedback results in coherence collapse for high currents [8], the lower power implies that isotropic feedback results in certain destructive effect. As $\theta_{\mathrm{p}}$ increases, the slope of the P-I curve increases from that in Fig. 2a. For $70^{\circ}<\theta_{p}<130^{\circ}$, the output power of the QDL with the polarization-rotated feedback is very close to or slightly higher than that of the solitary QDL. Figure $2 b$ depicts the power-current curves for $\theta_{\mathrm{p}}$ of $110^{\circ}$, where the total power with feedback is higher than that of the solitary QDL by a tiny amount. The corresponding threshold is $6.07 \mathrm{~mA}$, as shown in Fig. 2d. Note that the threshold is only reduced by $2.4 \%$ for large angle of rotation, whereas the threshold reduction is $7.2 \%$ for isotropic feedback. It was demonstrated experimentally that threshold current does not decrease when the polarization is rotated by $90^{\circ}$ (TM-mode feedback) for DFB lasers [29]. With a model taking both TE and TM modes into account, good agreement is achieved between theoretical and experimental results. Our results agree with the numerical work in [29] qualitatively. This implies that when feedback polarization makes a large angle with the original polarization, 
an orthogonal component in electric field is stimulated in the QDL.
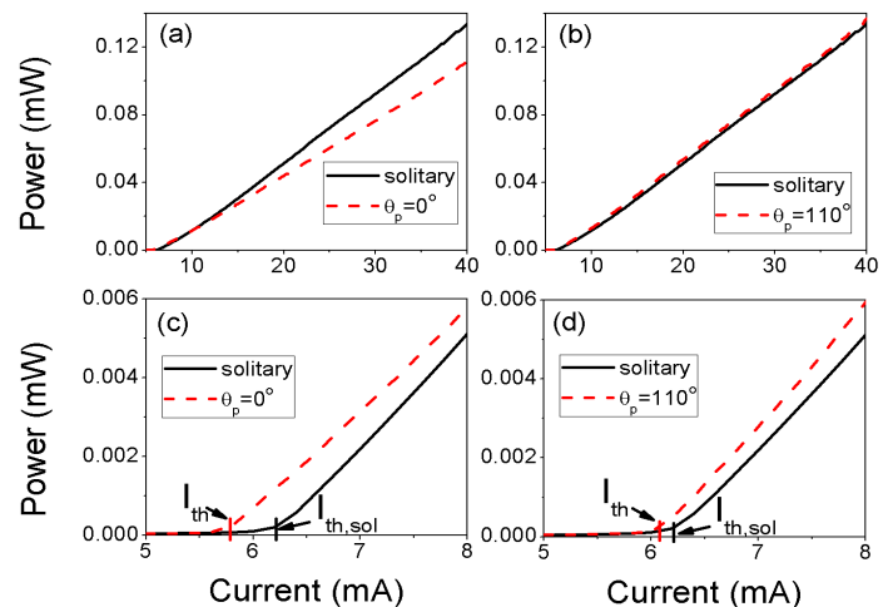

Fig. 2. Power-current curves of the solitary QDL and the QDL subject to (a) isotropic optical feedback, and (b) polarization-rotated feedback with $\theta_{p}=110^{\circ}$ as the bias current is increased to $40 \mathrm{~mA}$. (c) and (d) are corresponding curves zoomed in near threshold. The power reflectivity of the external cavity is $55.1 \%$

Polarization of the output beam is examined with a linear polarizer. The normalized transmitted power is plotted in Fig. 3 as a function of the orientation of the linear polarizer. The graph reveals that the QDL with isotropic feedback remains linearly polarized, and the polarization direction is the same as the solitary QDL. The minimum power for $\theta_{\mathrm{p}}$ of $110^{\circ}$ does not reach zero, and the angle at which the minimum power measured is shifted by $\sim 2^{\circ}$. The maximum power is achieved at the same angle as that of the solitary QDL. Hence the output is not linearly polarized when the QDL is subject to polarization-rotated optical feedback. The polarization of the output beam has a weak component approximately perpendicular to the dominant, horizontal component. During our investigation, strongest instabilities typically occur when $\theta_{\mathrm{p}}$ ranges from $108^{\circ}$ to $120^{\circ}$. Most data examples presented in this paper are for $\theta_{\mathrm{p}}$ of $108^{\circ}$ or $110^{\circ}$.

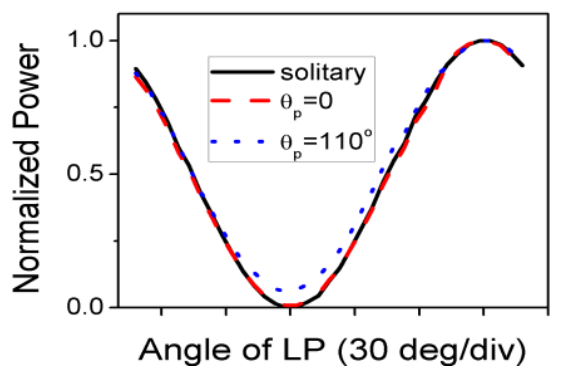

Fig. 3. Normalized power transmitting through the linear polarizer as the polarizer rotates through $180^{\circ}$ with respect to the horizontal direction. The bias current is $25 \mathrm{~mA}$.

The optical spectrum reveals that the free-running QDL operates in multiple longitudinal modes from the threshold. The mode spacing is $0.62 \mathrm{~nm}$. The number of modes increases with increasing bias current. Figure 4 illustrates optical spectra without and with optical feedback for two bias currents: Figs. $4 \mathrm{a}-4 \mathrm{c}$ are for $10 \mathrm{~mA}$, and Figs. $4 \mathrm{~d}-4 \mathrm{f}$ for $25 \mathrm{~mA}$. The envelope of modal peaks illustrates the shape of the spectral line. To evaluate the wavelength shift caused by optical feedback, we mark the mode at the center of the line shape. For $10 \mathrm{~mA}$, isotropic feedback makes the mode at the center shift to longer wavelength by $0.6 \mathrm{~nm}$ (Fig. 4b), whereas polarization-rotated feedback $\left(\theta_{\mathrm{p}}=108^{\circ}\right)$ causes a negligible shift (Fig. 4c). For 25 $\mathrm{mA}$, the red shift is $1.3 \mathrm{~nm}$ for $\theta_{\mathrm{p}}=0$ (Fig. $4 \mathrm{e}$ ) and $\underline{0.65} \mathrm{~nm}$ for $\theta_{\mathrm{p}}=108^{\circ}$ (Fig. 4f). The red shift caused by polarization-rotated feedback is $\underline{0.65} \mathrm{~nm}$ less than that caused by isotropic optical feedback. Hence, the red shift caused by polarization-rotated feedback is negligible for lower bias current and much less than that caused by isotropic feedback for higher bias current. The similar phenomenon was observed in a DFB laser, where the red shift caused by optical feedback of $\theta_{\mathrm{p}}=90^{\circ}$ is almost unnoticeable [잉. Figure $4 \mathrm{~g}(4 \mathrm{~h})$ illustrates a few modes around the center of the spectral line without and with isotropic feedback (rotated feedback of $\theta_{\mathrm{p}}=108^{\circ}$ ) for $25 \mathrm{~mA}$. Similar to the GS emission in [23], there is no spectral broadening in both Figs. $4 \mathrm{~g}$ and $4 \mathrm{~h}$.
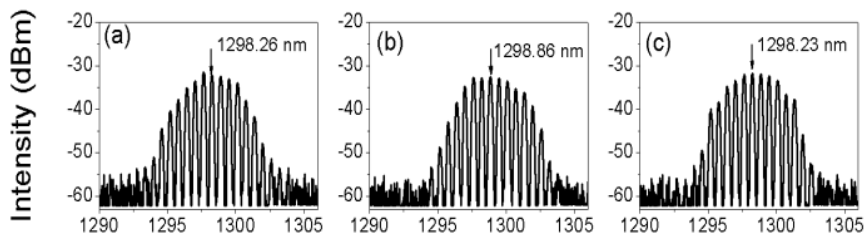

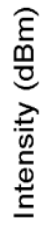
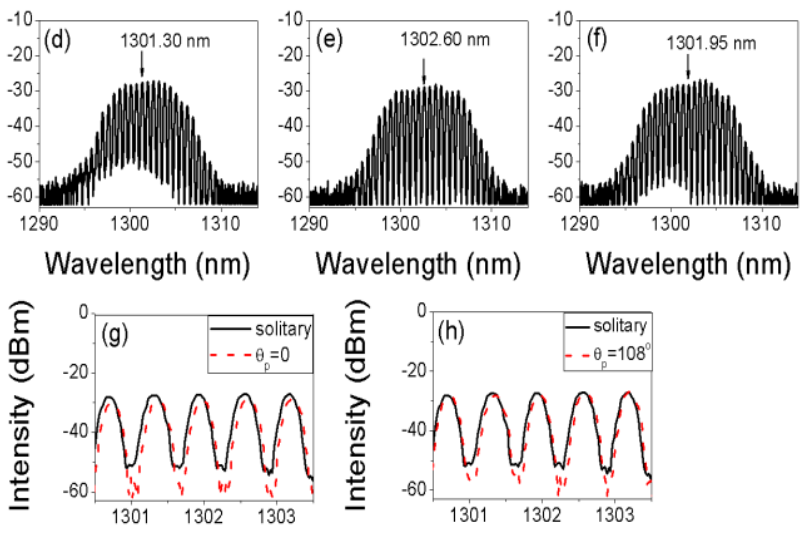

Wavelength $(\mathrm{nm})$

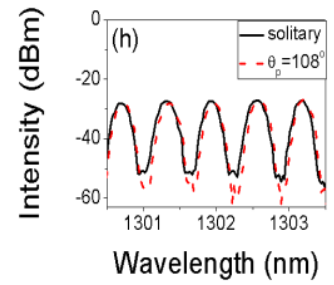

Fig. 4. Optical spectra of the QDL without and with optical feedback. Top row is for bias current of $10 \mathrm{~mA}$, middle and bottom row are for $25 \mathrm{~mA}$. (a) and (d): solitary QDL; (b) and (e): $\theta_{\mathrm{p}}=0$; (c) and (f): $\theta_{\mathrm{p}}=108^{\circ}$. The center mode is marked by the arrow labeled with its wavelength. $(\mathrm{g})$ and $(\mathrm{h})$ : zoomin views of optical spectra. The power reflectivity of the external cavity is $55.1 \%$. The length of the external cavity is $\sim 32 \mathrm{~cm}$.

\section{DYNAMICS INDUCED BY OPTICAL FEEDBACK}

Instabilities triggered by optical feedback are investigated using both power spectrum and time series. According to the manufacturer, the QDL is designed for data communications up to $2.5 \mathrm{Gbps}$, so its relaxation oscillation frequency is estimated around $1.3 \mathrm{GHz}$. But we were unable to observe the 
relaxation oscillation frequency in our experiment. Figure 5 gives power spectra of the QDL for $\theta_{\mathrm{p}}=0$ (isotropic feedback) and $\theta_{\mathrm{p}}=108^{\circ}$. The external cavity length is $\sim 32 \mathrm{~cm}$. Figures $5 \mathrm{a}$ and $5 \mathrm{~b}$ depict power spectra for the bias current of $22 \mathrm{~mA}$, and Figs. $5 \mathrm{c}$ and $5 \mathrm{~d}$ are for the bias current of $31 \mathrm{~mA}$. In Fig. 5a,
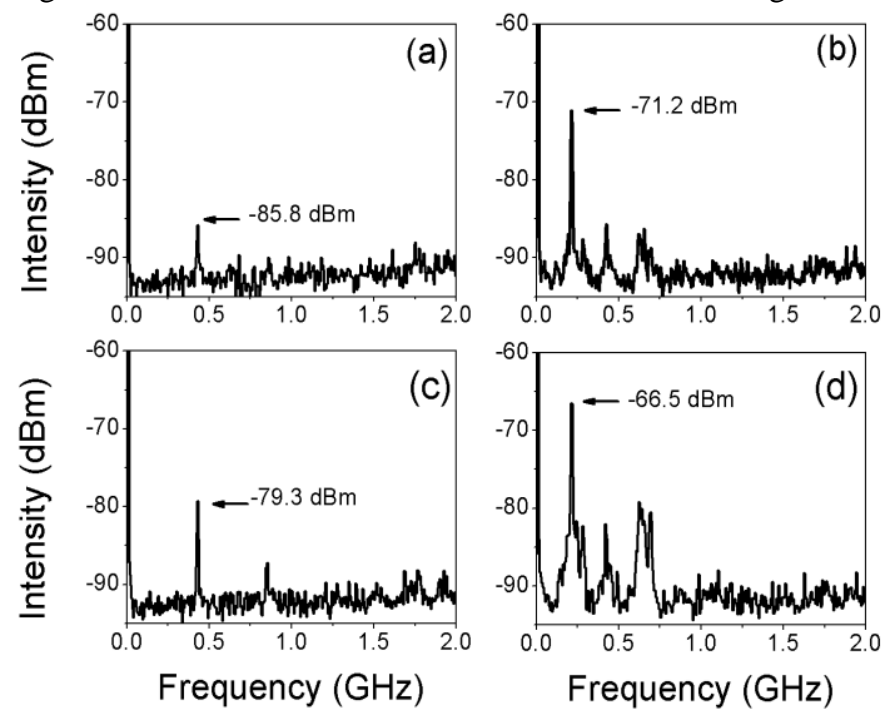

Fig. 5. Power spectra of the QDL subject to isotropic optical feedback (left column) and rotated feedback with $\theta_{\mathrm{p}}=108^{\circ}$ (right column). The bias current is $22 \mathrm{~mA}$ in (a) and (b) and $31 \mathrm{~mA}$ in (c) and (d). The external cavity length is around $32 \mathrm{~cm}$, and the reflectivity of the external cavity is $55.1 \%$.

the dynamic peak is located at $0.42 \mathrm{GHz}$, the external cavity resonance, $v_{\text {ext. }}$ It represents a periodic oscillation. In Fig. 5b, the strongest peak is located at $0.21 \mathrm{GHz}$, and the other two weaker peaks are around $0.42 \mathrm{GHz}$ and $0.63 \mathrm{GHz}$, respectively. They are the second and third harmonics of 0.21 GHz. This shows that the characteristic frequency becomes $0.21 \mathrm{GHz}$ for $\theta_{\mathrm{p}}=108^{\circ}$, one-half of that for isotropic feedback. Accordingly, the characteristic time is twice the round-trip time in the external cavity for polarization-rotated feedback. This is the same as in [34], where the period of square wave is triggered by polarization-rotated feedback is twice the roundtrip time in the external cavity. The RF peaks in Fig. $5 \mathrm{~b}$ are broader than that in Fig. 5a, implying that the oscillation is quasiperiodic. With a bias current of $31 \mathrm{~mA}$, the power spectrum of isotropic feedback (Fig. 5c) manifests sharp peaks at $0.42 \mathrm{GHz}$ and $0.84 \mathrm{GHz}$, and the intensity of the peak at $0.42 \mathrm{GHz}$ is $\underline{6.5} \mathrm{~dB}$ stronger than in Fig. 5a. Similarly, in Fig. $5 \mathrm{~d}$, the peaks at $0.21 \mathrm{GHz}$ and its higher order harmonics are stronger than those in Fig. 5b. Hence feedback dynamics is stronger with higher current as expected. For the same current, the peaks in the power spectra with rotated feedback are stronger and broader than those with isotropic feedback, meaning that the dynamics induced by polarization-rotated feedback is stronger and more complex.

Figure 6 gives the range of $\theta_{\mathrm{p}}$ in which the dynamics triggered by polarization-rotated feedback occurs for $25 \mathrm{~mA}$. As shown in Fig. 5, dynamics caused by polarization-rotated feedback is characterized by the dominant peak of the power spectrum located at $v_{\mathrm{ext}} / 2$, whereas dynamics induced by isotropic feedback is represented by the dominant peak located at $v_{\text {ext. }}$. The intensities of peaks located at $v_{\text {ext }}(0.42 \mathrm{GHz})$ and $v_{\text {ext }} / 2(0.21 \mathrm{GHz})$ are plotted as a function of $\theta_{\mathrm{p}}$. From $\theta_{\mathrm{p}}=0$ to $\theta_{\mathrm{p}}=90^{\circ}$, the dominant dynamical peak is at $0.42 \mathrm{GHz}$. The peak at $v_{\text {ext }} / 2$ appears from $100^{\circ}$ to $128^{\circ}$. Within this range, the peak at $v_{\text {ext }}$ is the second harmonic of the dominant peak. From $132^{\circ}$ to $180^{\circ}$, dominant peak occurs at $v_{\text {ext }}$. Thus, the dynamics characterized by $v_{\mathrm{ex}} / 2$ occurs when the polarization of feedback beam makes a large angle with the horizontal direction. The dynamics characterized by $v_{\text {ext }} / 2$ is only observed for $\theta_{\mathrm{p}}$ located in the second quadrant of the xy plane. It does not happen symmetrically about the y-direction. The reason for the asymmetry is unclear and may be attributed to some internal parameters of the QDL. In Fig. 6 the strongest peak is observed when $\theta_{\mathrm{p}}$ is $120^{\circ}$. Note that the strongest RF peak does not always occur at $\theta_{\mathrm{p}}=120^{\circ}$; it typically occurs for a value of $\theta_{\mathrm{p}}$ ranging between $108^{\circ}$ and $120^{\circ}$ when the bias current is $25 \mathrm{~mA}$. This variation can be attributed to the slight, day-to-day variation in the experimental setup. In addition, the range in which the strongest RF peak is obtained can vary slightly for different bias currents.

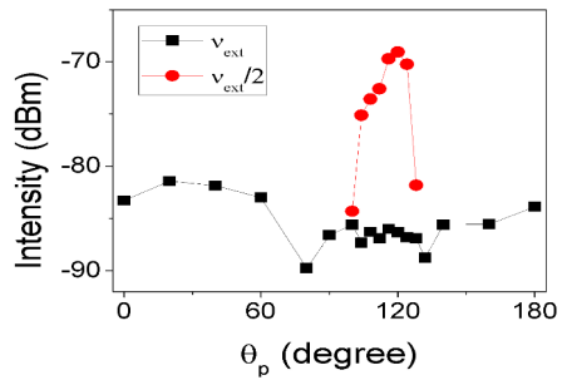

Fig. 6. Intensity of RF peaks in the power spectra versus the polarization angle. The bias current is $25 \mathrm{~mA}$, the external cavity length is $\sim 32 \mathrm{~cm}$, and the reflectivity of the external cavity is $55.1 \%$.

The effect of bias current on feedback dynamics is demonstrated in Fig. 7, where the intensity of the strongest RF peak in the power spectrum is plotted against the bias current. The strongest peak in the power spectrum is located at 0.42 $\mathrm{GHz}$ for $\theta_{\mathrm{p}}=0^{\circ}$ and $0.21 \mathrm{GHz}$ for $\theta_{\mathrm{p}}=108^{\circ}$. As shown, dynamics induced by isotropic feedback does not occur until $18 \mathrm{~mA}$, which is 2.9 times the threshold of the solitary QDL, $\mathrm{I}_{\mathrm{th}, \mathrm{sol}}$. This agrees with previous results that quantum dot lasers are less sensitive to optical feedback than their bulk or quantum-well counterparts [16]-[22]. Dynamics triggered by rotated feedback occurs from $11 \mathrm{~mA}\left(\mathrm{I}=1.8 \mathrm{I}_{\mathrm{th}}, \mathrm{sol}\right)$, a current much lower than the lowest current for isotropic feedback. With the same bias current, the dynamic peak caused by rotated feedback is $11.6 \mathrm{~dB}$ to $16.5 \mathrm{~dB}$ stronger than that for isotropic feedback. This shows that the QDL is much more sensitive to polarization-rotated optical feedback. 


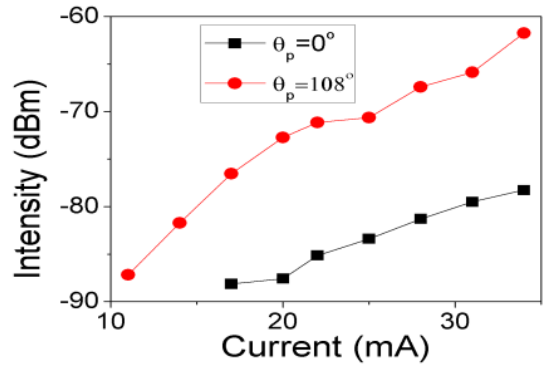

Fig. 7. Intensity of the strongest peak in the power spectra versus bias current. The external cavity length is $\sim 32 \mathrm{~cm}$, and the reflectivity of the external cavity is $55.1 \%$.

Feedback strength is another important parameter for dynamics caused by optical feedback [8]. In our experiment, feedback strength is adjusted by changing the power reflectivity of the external cavity, $R_{\text {ext }}$, where $R_{\text {ext }}$ depends on the reflectance of the non-polarizing beamsplitter, transmittance of the neutral density filter, and reflectance of the end mirror. The intensity of the strongest RF peak in the power spectrum depends on $\mathrm{R}_{\text {ext }}$, as depicted in Fig. 8. While isotropic feedback dynamics is triggered for $R_{\text {ext }}>24 \%$, polarization-rotated feedback results in dynamics for $R_{\text {ext }}$ $>17 \%$. For the same value of $\mathrm{R}_{\text {ext }}$, the intensity of the peak caused by polarization-rotated feedback is much higher than that caused by isotropic feedback: the intensity difference ranges from $11.1 \mathrm{~dB}$ to $14.3 \mathrm{~dB}$. This confirms that the QDL is more sensitive to optical feedback when the polarization of feedback beam is rotated a large angle from the original polarization.

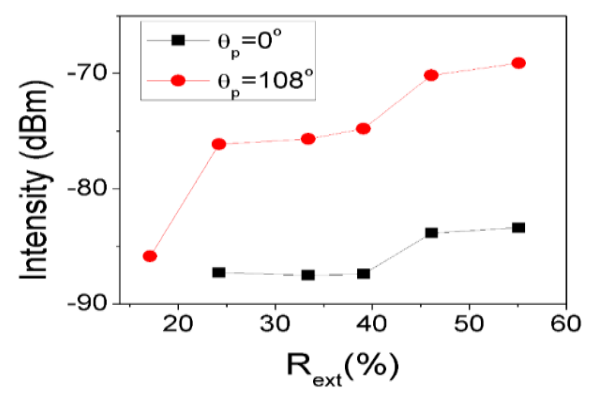

Fig. 8. Intensity of the strongest peak in the power spectra versus power reflectivity, $R_{\text {ext. }}$ The bias current is $25 \mathrm{~mA}$, and the length of external cavity is $\sim 32 \mathrm{~cm}$.

Given that the output beam is not perfectly linearly polarized when subject to feedback of large-angle rotation, polarization dynamics triggered by rotated feedback is investigated by sending the output beam through the linear polarizer (LP). The angle between the axis of the LP and the $\mathrm{x}$ axis, $\alpha$, is measured counterclockwise from the $\mathrm{x}$-axis to the axis of the LP. As shown in Fig. 3, the transmitted power reaches maximum for $\alpha=0$ or $180^{\circ}$ and minimum for $\alpha$ around $90^{\circ}$. Fig. 9 illustrates the intensity of the RF peak located at $v_{\text {ext }} / 2$ as a function of $\alpha$. The weakest RF peaks is obtained around $90^{\circ}$, which is expected because the output has minimum power around this angle. The strongest dynamics, however, does not occur when the LP is parallel to the $\mathrm{x}$-axis. Instead, local maxima of the RF peak are obtained around $45^{\circ}$ and $135^{\circ}$, respectively. Note that these two directions are perpendicular to each other.

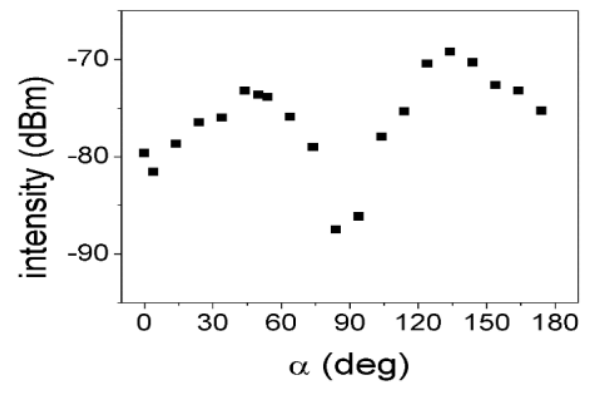

Fig. 9. The intensity of the RF peak located at $v_{\text {ext }} / 2$ as a function of $\alpha$. The experimental parameters are $\theta_{\mathrm{p}}=108^{\circ}, \mathrm{I}=25 \mathrm{~mA}, \mathrm{R}_{\mathrm{ext}}=55.1 \%$, and $\mathrm{L}$ is $\sim 32$ $\mathrm{cm}$.

Interestingly, the RF peak of the polarized beam can be stronger than its counterpart of the total power. Figure 10 compares the power spectra and time series of the total power to those of the polarized light. Figures $10 \mathrm{a}$ to $10 \mathrm{c}$ are power spectra, and Figs. 10d to 10f are corresponding time series. The intensity of the dominant RF peak is $-74.8 \mathrm{dBm}$ for total

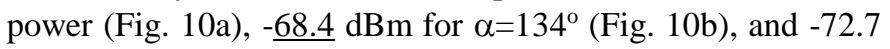
$\mathrm{dBm}$ for $\alpha=44^{\circ}$ (Fig. 10c). The temporal fluctuations with the largest peak-to-peak amplitudes (Fig. 10e) correspond to the strongest RF peak (Fig. 10b). The time series reveal that the fluctuations in Fig. 10e drop down whereas the fluctuations in Fig. 10f burst up. Hence it is plausible to conjecture that the fluctuations in these two polarized lights may be anticorrelated.
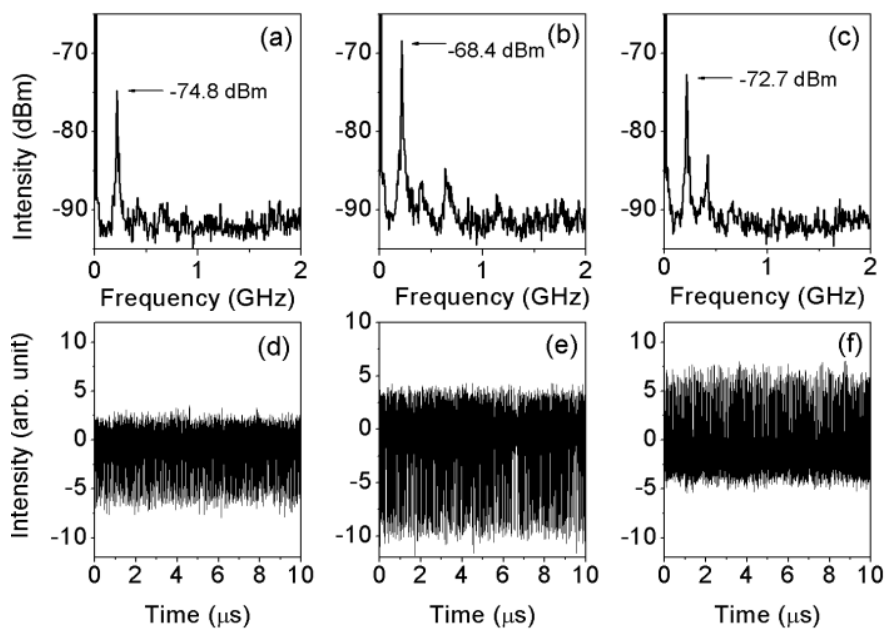

Fig. 10. (a) to (c) RF spectrum and (d) to (f) corresponding time series for $\mathrm{I}=25 \mathrm{~mA}, \theta_{\mathrm{p}}=120^{\circ}$, and $\mathrm{L} \sim 32 \mathrm{~cm}$. (a) and (d) are for total power, (b) and (e) are for $\alpha=134^{\circ}$, and (c) and (f) for $\alpha=44^{\circ}$.

To verify this conjecture, polarization-resolved measurements are needed. For this purpose, we introduced a half-wave plate to rotate the incident beam and a polarizing beamsplitter (PBS) to divide the beam into a horizontal component and vertical component (not shown in Fig. 1). Note that the incident beam is dominantly polarized along $\mathrm{x}$-axis. When it is rotated an angle $\alpha$ from the $\mathrm{x}$-axis, the light passing through the polarizing beamsplitter will be the projection of the rotated beam in the horizontal direction. Named as $\mathrm{x}$ - 
component, this is equivalent to the light passing through a linear polarizer whose axis makes an angle of $\alpha$ with the $\mathrm{x}$ axis. The corresponding vertical component, named $y$ component, is reflected off the PBS. The y-component is equivalent to the light passing through the linear polarizer that makes angle of $\alpha \pm 90^{\circ}$ with the $\mathrm{x}$-axis, where "+" is for $\alpha<90^{\circ}$ and "-" for $\alpha>90^{\circ}$. Either component is sent to a fiber coupler that can be connected to a fast detector (New Focus 1554-B$50,12 \mathrm{GHz}$ ), which allows us to measure dynamics of the two components simultaneously.

Polarization resolved power-current curves are illustrated in Fig. 11, for which the value of $\alpha$ is set at zero. The output beam is linearly polarized along the $\mathrm{x}$-direction in the whole current range for the free-running QDL (Fig. 11a) and the QDL with isotropic feedback (Fig. 11b). For $\theta_{\mathrm{p}}=110^{\circ}$ (Fig.
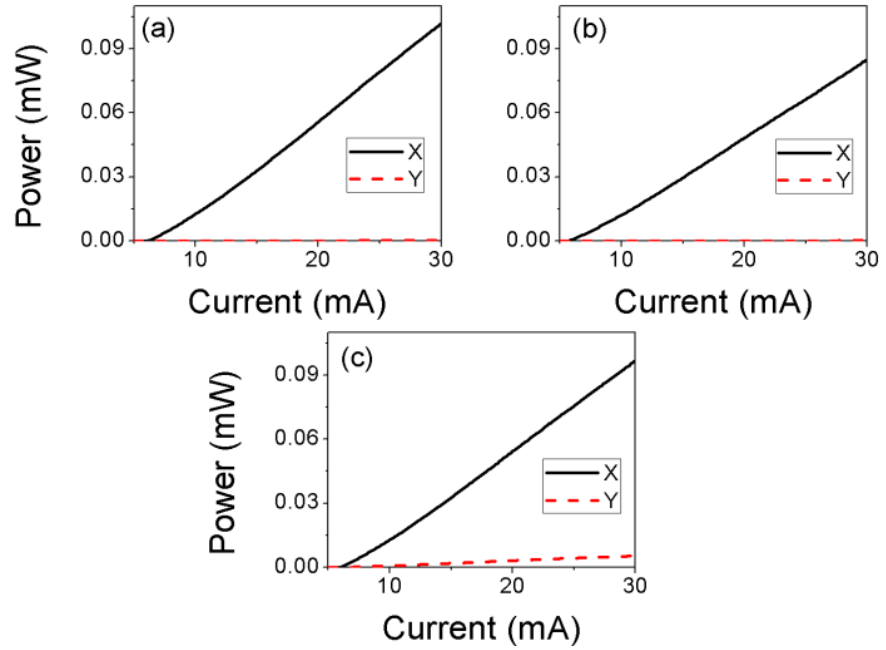

Fig. 11. Polarization-resolved power-current curves of (a) the solitary QDL, (b) the QDL subject to isotropic optical feedback, and (c) the QDL subject to rotated feedback of $\theta_{p}=110^{\circ}$. The reflectivity of the external cavity is $55.1 \%$.

11c), a dominant x-polarized power and a weak orthogonal component are obtained, very similar to the numerical result for TM feedback [30].
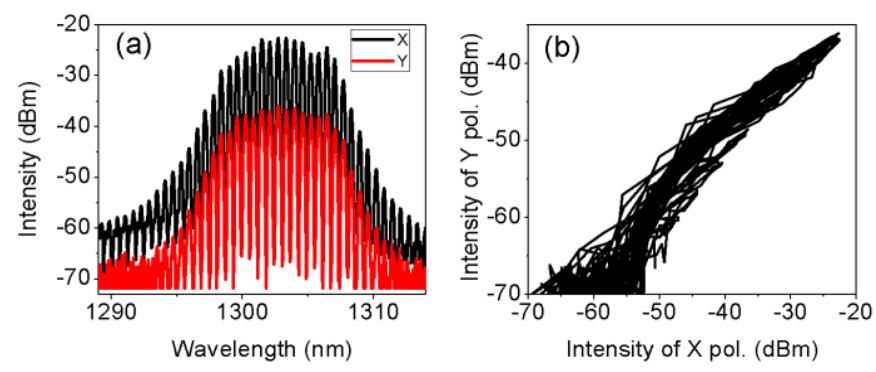

Fig. 12. (a) Polarization-resolved optical spectra of the QDL subject to optical feedback with $\theta_{\mathrm{p}}$ of $110^{\circ}$. (b) Intensity of the Y-polarized optical spectrum versus that of the X-polarized spectrum. The bias current is $25 \mathrm{~mA}$, and $\mathrm{R}_{\mathrm{ext}}$ is $55.1 \%$.

Polarization resolved optical spectra are illustrated in Fig. 12a. While the y-component is overall much weaker than the $\mathrm{x}$-component, y-polarized modal powers do not decrease by the same amount. More specifically, y-polarized modes are typically 11 to $14 \mathrm{~dB}$ weaker than the corresponding $\mathrm{x}$ - polarized modes. Thus, modal polarization states are not the same. Fig. 12b plots y-polarized spectrum versus x-polarized one. A curved relation is obtained. This indicates that the $y-$ polarized modal power is not purely reflection. In other words, a weak y-polarized component is stimulated by the polarization-rotated optical feedback.

The cross correlation function is calculated for time signals whose polarizations are orthogonal to each other. The time series were taken simultaneously. When the time delay is zero, the correlation function has the most negative value. One example is shown in Fig. 13, where $\alpha$ is $136^{\circ}$ and $\theta_{\mathrm{p}}=110^{\circ}$. The time series of the $\mathrm{x}$ - and $\mathrm{y}$-polarizations are depicted in Fig. 13a. It is obvious that the two fluctuations are anticorrelated. The correlation function reaches -0.846 when the time delay is zero, which is the minimum value of the function. This confirm the conjecture that the dynamics along directions of $46^{\circ}$ and $136^{\circ}$ from the $\mathrm{x}$-axis are anticorrelated. The dynamics of the total power is weaker than those in the $136^{\circ}$ - and $46^{\circ}$-directions because the temporal fluctuations in these two directions cancel each other partially. The correlation function also manifests a damped oscillation as the time series have larger relative time lags (Fig. 13b). The period of the oscillation is $\sim 5 \mathrm{~ns}$, close to the reciprocal of $0.21 \mathrm{GHz}$.
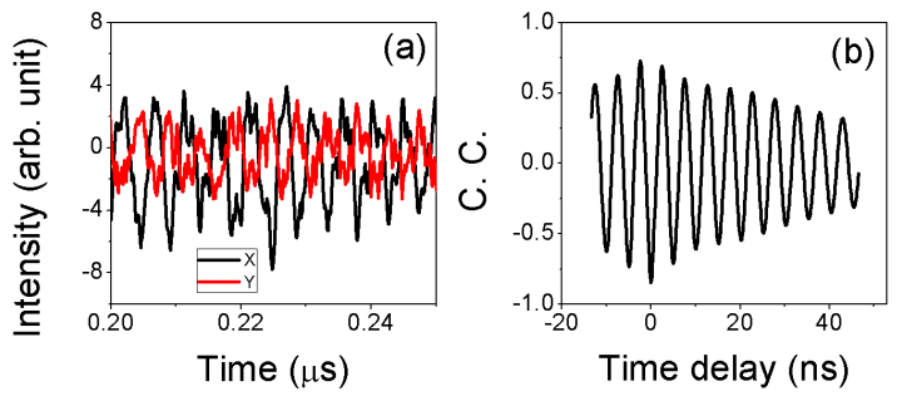

Fig. 13. (a) Time series of $x$ - and y-polarizations and (b) their cross correlation when $\alpha$ is $136^{\circ}$ and $\theta_{\mathrm{p}}$ is $110^{\circ}$. The other experimental parameters are $\mathrm{I}=25 \mathrm{~mA}, \mathrm{R}_{\mathrm{ext}}=55.1 \%$, and $\mathrm{L} \sim 32 \mathrm{~cm}$.

The value of the minimum cross correlation is plotted as a function of $\alpha$ in Fig. 14. The graph is approximately symmetric around $\alpha=90^{\circ}$, since the data in the range of $\alpha>90^{\circ}$ just switches the $\mathrm{x}$ - and y-components for $\alpha<90^{\circ}$. For $34^{\circ}<$ $\alpha<71^{\circ}$ and $125^{\circ}<\alpha<157^{\circ}$, the correlation is less than -0.8 . Comparing this to Fig. 9, the strongest polarization dynamics occurs in the above regions. This explains why the RF peak of the light transmitting through the linear polarizer can be stronger than the RF peak in a certain range of $\alpha$. For $\alpha$ of 0 and $90^{\circ}$, the value of cross correlation is positive but less than 0.18 , indicating that the horizontal and vertical components of the total power are almost not correlated. The low correlation is attributed to the weak power and weak dynamics in the ycomponent. The value of the minimum correlation is not the same for $\alpha=0$ and $\alpha=90^{\circ}$ because the time series used for calculation were taken at different instants. Accordingly, the correlation between the $\mathrm{x}$ - and $\mathrm{y}$-components may vary slightly with different trials. 


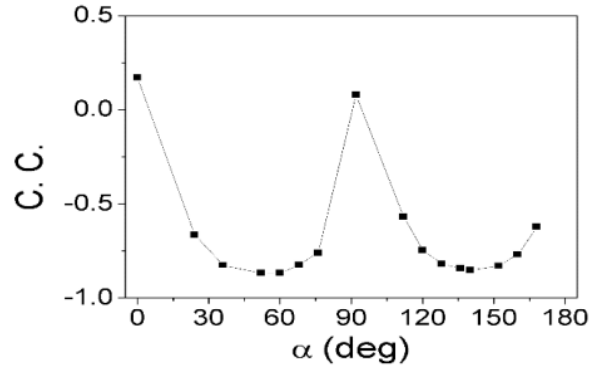

Fig. 14. Minimum cross correlation between the $\mathrm{x}$ - and $\mathrm{y}$-components versus $\alpha$. The experimental parameters are $\mathrm{I}=25 \mathrm{~mA}, \theta_{\mathrm{p}}=110^{\circ}, \mathrm{R}_{\mathrm{ext}}=55.1 \%$, and $\mathrm{L} \sim$ $32 \mathrm{~cm}$.

For multimode QDLs, it has been shown that modal intensities can oscillate chaotically but obey a highly organized antiphase dynamics leading to a constant total power [26]. One can expect that polarization dynamics of each mode may be different from that of the total power. Without instruments for mode selection, we used polarization-resolved optical spectra to extract some information. Given that polarization of the total power with rotated feedback is still dominantly $\mathrm{x}$ polarized, when the beam is rotated an angle of $46^{\circ}$ or $136^{\circ}$ by the half-wave plate, its power in the x-direction and the $y$ direction should be essentially the same. Polarization-resolved optical spectra for $\alpha$ of $136^{\circ}$ is depicted in Fig. 15a, where the $\mathrm{x}$-component is equivalent to the optical spectrum when the light transmits the linear polarizer that makes an angle of $136^{\circ}$ with the $\mathrm{x}$-axis, and the $\mathrm{y}$-component corresponds to the transmitted optical spectrum when the linear polarizer is $46^{\circ}$ away from the $\mathrm{x}$-axis. The modal powers in the $\mathrm{x}$ - and $\mathrm{y}$ spectra are very similar except the modes around the center wavelength. The optical spectra are zoomed in in Fig. 15b. Several modes in the $\mathrm{x}$-component are 2 to $3 \mathrm{~dB}$ stronger than their counterparts in the y-component. In linear scale, the powers of these modes are 1.58 to 2 times the power of their $y$ counterparts. This shows that modal powers in the $\mathrm{x}$ component and $y$-component are considerably different for some strongest modes. It is possible that different modal polarizations contribute to the observed anticorrelated polarization dynamics, which results in weaker dynamics in the total power. Relative phase between longitudinal modes is another important factor for dynamics.
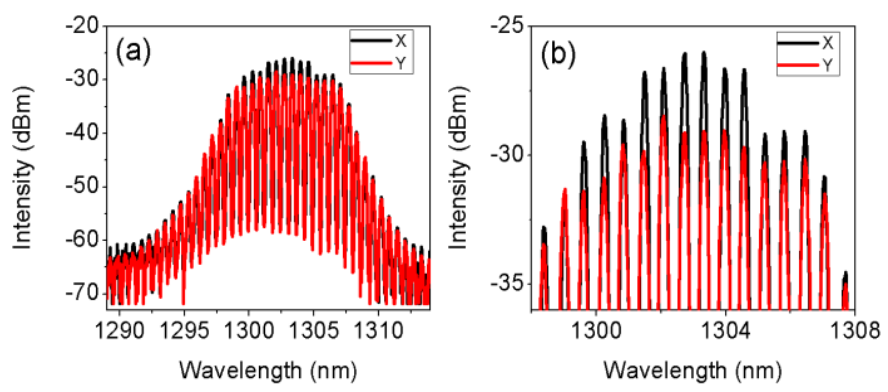

Fig. 15. (a) Polarization resolved optical spectra of the $x$ - and $y$-component for $\alpha=136^{\circ}$. (b) Modes around the center wavelength. I=25 mA, $\theta_{p}=110^{\circ}$, $\mathrm{R}_{\mathrm{ext}}=55.1 \%$, and $\mathrm{L}$ is $\sim 32 \mathrm{~cm}$.

\section{DISCUSSION AND CONCLUSION}

Previous research has demonstrated that polarizationrotated optical feedback can lead to chaotic fluctuations [14], [31]-[33] and conceal time delay features simultaneously [35], [36], which will be useful in applications involving optical chaos. We have studied dynamics of a multimode QDL with polarization-rotated feedback. The P-I curves of the total power and for $\mathrm{x}$ - and $\mathrm{y}$-polarizations agree with the results in [30] for DFB lasers, indicating that a weak orthogonal polarization is stimulated in the QDL. Our results show that when the polarization of feedback beam is rotated $100^{\circ}$ to $128^{\circ}$ away from the original polarization, instabilities occur at lower current and weaker feedback strength than those for isotropic feedback. This shows that a QDL is more sensitive to optical feedback when the polarization of feedback beam rotates through a large angle. In addition, the induced dynamics demonstrates stronger and broader RF peaks in power spectra. A large angle between feedback polarization and the $\mathrm{x}$ direction means that feedback has a stronger y-component and in turn induces a weak y-polarization in laser output. The observed dynamics may be related to competition between the $\underline{x-}$ and $y$-polarizations for charge carriers. The relation between dynamics and feedback strength in our experiment differs from the observations in a DFB laser, in which stronger optical feedback is needed in order to observe instabilities for polarization-rotated feedback [30]. This difference can be attributed to different active media in the DFB and the QDL. Theoretical analysis has shown that external cavity modes (ECMs) affect feedback sensitivity of QDLs subject to isotropic feedback [21]. Polarization-rotated optical feedback may alter the stability boundary of ECMs and in turn increase feedback sensitivity. Since the stability of QDLs emitting in ES state can be comparable to the quantum-well lasers [23], [24], it is plausible to conjecture that polarization-rotated feedback may trigger more complex or chaotic dynamics in a QDL emitting in ES state. However, our laser is in GS emission in the whole current range, so we were unable to check this conjecture. A study on QDLs in ES emission with polarization-rotated feedback may reveal interesting results. Because our QDL operates with many longitudinal modes, modal dynamics will be an important part for obtaining a complete picture of the observed dynamics and is worthy to explore for interested researchers. A study on modal polarization dynamics will help understanding the anticorrelated fluctuations in orthogonally polarized components.

In conclusion, a multimode QDL is more sensitive to polarization-rotated optical feedback than to isotropic feedback. Polarization dynamics can be significantly stronger than the dynamics in the total power, which is caused by anticorrelated fluctuations of orthogonally polarized components. The anticorrelated fluctuations may be related to modal dynamics. This first experimental investigation reveals interesting phenomena and leaves open questions for further study both experimentally and theoretically. 


\section{REFERENCES}

[1] A. E. Zhukov, M. V. Maksimov, and A. R. Kovsh, "Device characteristics of long-wavelength lasers based on self-organized quantum dots," Semiconductors, vol. 46, no. 10, pp. 1225-1250, Oct. 2012.

[2] C. Ribbat, R. L. Sellin, I. Kaiander, F. Hopfer, N. N. Ledentsov, and D. Bimberg, "Complete suppression of filamentation and superior beam quality in quantum-dot lasers," Appl. Phys. Lett., vol. 82, no. 6, pp. 952-954, Feb. 2003.

[3] D. Bimberg, "Quantum dot based nanophotonics and nanoelectronics," Electron. Lett., vol. 44, no. 3, pp. 168-171, Jan. 2008

[4] A. Markus, J. X. Chen, C. Paranthoen, and A. Fiore, "Simultaneous two-state lasing in quantum-dot lasers," Appl. Phys. Lett., vol. 82, no. 12, pp. 1818-1820, Mar. 2003.

[5] J. H. Osmundsen and N. Gade, "Influence of optical feedback on laser frequency spectrum and threshold conditions," IEEE J. Quantum Electron., vol. QE-19, no. 3, pp. 465-469, Mar. 1983.

[6] J. Mørk, B. Tromborg, and P. L. Christiansen, "Bistability and lowfrequency fluctuations in semiconductot lasers with optical feedback: a theoretical analysis," IEEE J. Quantum Electron., vol. 24, no. 2, pp. 123-129, Feb. 1988.

[7] T. Mukai and K. Otsuka, "New route to optical chaos: successivesubharmonic-oscillation cascade in a semiconductor laser coupled to an external cavity," Phys. Rev. Lett., vol. 55, no. 17, pp. 1711-1714, Oct. 1985.

[8] T. Heil, I. Fischer, and W. Elsäßer, "Coexistence of low-frequency fluctuations and stable emission on a single high-gain mode in semiconductor lasers with optical feedback," Phys. Rev. A, vol. 58, no. 4, R2672-R2675, Oct. 1998

[9] S. Jiang, Z, Oabm N, Dagenais, R. A. Morgan, and K. Kojima, "Influence of external optical feedback on threshold and spectral characteristics of vertical-cavity surface-emitting lasers," IEEE Photon. Technol. Lett., vol. 6, no. 1, pp. 34-36, Jan. 1994.

[10] J. Y. Law and G. P. Agrawal, "Effects of optical feedback on static and dynamic characteristics of vertical-cavity surface-emitting lasers," IEEE J. Sel. Top. Quantum Electron., vol. 3, no. 2, pp. 353-358, Apr. 1997.

[11] M. Giudici, S. Balle, T. Ackemann, S. Barlands, and J. R. Tredicce, "Polarization dynamics in vertical-cavity surface-emitting lasers with optical feedback: experiment and model," J. Opt. Soc. Am. B, vol. 16, no. 11, pp. 2114-2123, Nov. 1999

[12] M. Sondermann, H. Bohnet, and T. Ackemann, "Low-frequency fluctuations and polarization dynamics in vertical-cavity surfaceemitting lasers with isotropic feedback," Phys. Rev. A, vol. 67, no. 2, Art. No. 021802, Feb. 2003.

[13] A. Argyris, D. Syvridis, L. Larger, V. Annovazzi, I. Fischer, J. GarciaOjalvo, C. R. Mirasso, L. Pesquera, and K. A. Shore, "Chaos-based communications at high bit rates using commercial fibre-optic links," Nature, vol. 438, no. 17, pp. 343-346, Nov. 2005.

[14] N. Oliver, M. C. Soriano, D. W. Sukow, and I. Fischer, "Dynamics of a semiconductor laser with polarization-rotated feedback and its utilization for random bit generation," Opt. Lett., vol. 36, no. 23, pp. 4632-4634, Dec. 2011

[15] J.-P. Zhuang and S.-C. Chan, "Tunable photonic microwave generation using optically injected semiconductor laser dynamics with optical feedback stabilization," Opt. Lett., vol. 38, no. 3, pp. 344-346, Feb. 2013.

[16] D. O'Brien, S. P. Hegarty, G. Huyet, J. G. McInerney, "Feedback sensitivity of $1.3 \mu \mathrm{m} \mathrm{InAs/GaAs} \mathrm{quantum} \mathrm{dot} \mathrm{lasers,"} \mathrm{Electron.} \mathrm{Lett.,}$ vol. 39, no. 25, pp. 1819-1820, Dec. 2003

[17] G. Huyet, D. O’Brien, S. P. Hegarty, J. G. McInerney, A. V. Uskov, D. Bimberg, C. Ribbat, V. M. Ustinov, A. E. Zhukov, S. S. Mikhrin, A. R. Kovsh, J. K. White, K. Hinzer, and A. J. SpringThorpe, "Quantum dot semiconductor laser with optical feedback," Phys. Stat. Sol. A, vol. 201, no. 2, pp. 345-352, Jan. 2004.

[18] O. Carroll, I. O’Briscoll, S. P. Hegarty, G. Huyet, H. Houlihan, E. A. Viktorov, and P. Mandel, "Feedback induced instabilities in a quantum dot semiconductor laser," Opt. Express, vol. 14, no. 22, pp. 1083110837, Oct. 2006.

[19] D. O'Brien, S. P. Hegarty, G. Huyet, and A. V. Uskov, "Sensitivity of quantum-dot semiconductor lasers to optical feedback," Opt. Lett., vol. 29, no. 10, pp. 1072-1074, May 2004.
[20] E. A. Viktorov, P. Mandel, and G. Huyet, "Long cavity quantum dot lasers," Opt. Lett., vol. 32, no. 10, pp. 1268-1270, May 2007.

[21] C. Otto, K. Lüdge, and E. Schöll, "Modeling quantum dot lasers with optical feedback: sensitivity of bifurcation scenarios," Phys. Status Solidi B, vol. 247, no. 4, pp. 829-845, Jan. 2010.

[22] B. Globisch, C. Otto, E. Schöll, and K. Lüdge, "Influence of carrier lifetimes on the dynamical behavior of quantum-dot lasers subject to optical feedback," Phys. Rev. E, vol. 86, no. 4, Art. No. 046201, Oct. 2012.

[23] H. Huang, D. Arsenijević, K. Schires, T. Sadeev, D. Bimberg, and F. Grillot, "Multimode optical feedback dynamics of InAs/GaAs quantumdot lasers emitting on different lasing states," AIP Advances, vol. 6, no. 12, Art. No. 125114, Dec. 2016.

[24] H. Huang, L.-C. Lin, C.-Y. Chen, D. Arsenijević, D. Bimberg, F.-Y. Lin, and F. Grillot, "Multimode optical feedback dynamics of InAs/GaAs quantum-dot lasers emitting exclusively on ground or excited states: transition from short- to long-delay regimes," Opt. Express, vol. 26, no. 2, pp. 1743-1751, Jan. 2018.

[25] H. Huang, J. Duan, D. Jung, A. Y. Liu, Z. Zhang, J. Norman, J. E. Bowers, and F. Grillot, "Analysis of the optical feedback dynamics in InAs/GaAs quantum dot lasers directly grown on silicon," J. Opt. Am. B, vol. 35, no. 11, pp. 2780-2787, Nov. 2018.

[26] Y. Tanguy, J. Houlihan, G. Huyet, E. A. Vikorov, and P. Mandel, "Synchronization and clustering in a multimode quantum dot laser," Phys. Rev. Lett., vol. 96, no. 5, Art. No. 053902, Feb. 2006.

[27] Y. Hong, P. S. Spencer, and K. A. Shore, "Suppression of polarization switching in vertical-cavity surface-emitting lasers by use of optical feedback," Opt. Lett., vol. 29, no. 18, pp. 2152-2153, Sep. 2004.

[28] G. Giacomelli, F. Marin, and M. Romanelli, "Multi-time-scale dynamics of a laser with polarized optical feedback," Phys. Rev. A, vol. 67, no. 5, Art. No. 053809, May 2003.

[29] H. Lin, J. Hoshue, Z. J. Lapin, and A. Valle, "Polarization instabilities in a multi-transverse-mode vertical-cavity surface-emitting laser with polarized optical feedback," Opt. Commun., vol. 283, no. 7, pp. 14241433, Apr. 2010.

[30] T. Heil, A. Uchida, D. Davis, and T. Aida, "TE-TM dynamics in a semiconductor laser subject to polarization-rotated optical feedback," Phys. Rev. A, vol. 68, no. 3, Art. No. 033811, Sep. 2003.

[31] D. W. Sukow, A. Gavrielides, T. Erneux, M. J. Baracco, Z. A. Parmenter, and K. L. Blackburn, "Two-field description of chaos synchronization in diode lasers with incoherent optical feedback and injections," Phys. Rev. A, vol. 72, no. 4, Art. No. 043818, Oct. 2005.

[32] N. Shibasaki, A. Uchida, S. Yoshimori, and P. Davis, "Characteristics of chaos synchronization in semiconductor lasers subject to polarization-rotated optical feedback," IEEE J. Quantum Electron., vol. 42, no. 3, pp. 342-350, Mar. 2006.

[33] Y. Takeuchi, R. Schogenji, and J. Ohtsubo, "Chaotic dynamics in semiconductor lasers subjected to polarization-rotated optical feedback," Appl. Phys. Lett., vol. 93, no. 18, Art. No. 181105, Nov. 2008.

[34] A. Gavrielides, T. Erneux, D. W. Sukow, G. Burner, T. McLachlan, J. Miller, and J. Amonette, "Square-wave self-modulation in diode lasers with polarization-rotated optical feedback," Opt. Lett., vol. 31, no. 13, pp. 2006-2008, Jul. 2006

[35] P. Xiao, Z.-M. Wu, J.-G. Wu, L. Jiang, T. Deng, X. Tang, L. Fan, and G.-Q. Xia, "Time-delay signature concealment of chaotic output in a vertical-cavity surface-emitting laser with double variable-polarization optical feedback," Opt. Commun., vol. 286, pp. 339-343, Jan. 2013.

[36] S. Priyadarshi, Y. Hong, I. Pierce, and K. A. Shore, "Experimental investigation of time-delay signature concealment in chaotic external cavity VCSELs subject to variable optical polarization angle of feedback," IEEE J. Sel. Top. Quantum Electron., vol. 19, no. 4, Art. No. 1700707, Jul./Aug. 2013

Hong Lin did her undergraduate study in Beijing University of Aeronautics and Astronautics (also known as Beihang University) and Nankai Univerisity. She received the B.S. degree in physics in 1982 and M.S. degree in physics in 1986 from Beihang University, China, and Ph.D. degree in physics from Bryn Mawr College, USA. in 1991. She was a lab instructor in 1982-83 and a lecturer in 1986 with Beihang University. In 1991 she joined the Department of Physics and Astronomy at Bates College, U.S.A., where she became an 
Assistant Professor in 1991, an Associate Professor in 1998, and a Professor since 2004. She was the Chair of the Division of Natural Sciences and Mathematics, Bates College from 2007 to 2011 and the Chair of the Department of Physics and Astronomy, Bates College from 2014 to 2018. Her research interests are nonlinear dynamics and their applications in gas lasers, vertical-cavity surface-emitting lasers, quantum dot lasers, and photorefractive devices.

Prof. Lin is a senior member of the Optical Society of America.

Yanhua Hong received the B.Sc. degree in physics from Fujian Normal University, Fuzhou, China, the M.S. degree in physics from Beijing Normal University, Beijing, China, and the Ph.D. degree in optics from the Institute of Physics, Chinese Academy of Sciences, Beijing, in 1987, 1990, and 1993, respectively. She was a Lecturer with Beihang University, Beijing, from 1993 to 1997. Since 1997, she has been with Bangor University, Bangor, U.K., where she became a Permanent Research Staff in 2007, Lecturer in 2013, and Senior Lecturer in 2016. She is the author or co-author of more than 200 journal and conference papers. Her current research interests include microwave photonics, semiconductor laser dynamics, and digital signal processing for high-speed optical communications.

Salim Ourari received his B.S. degree in physics from Bates College, Lewiston, ME, USA in 2018. He is currently a graduate student with the Department of Electrical Engineering, Princeton University.

Tianyao Huang received his B. S. degree in physics and mathematics from Bates College, Lewiston, ME, USA in 2019. He is currently a graduate student with the Department of Electrical Engineering, Northwestern University.

Chen Yang is a physics major at Bates College, Lewiston, ME, USA. 